\title{
Historique et but des recommandations formalisées d'experts communes à deux sociétés (SFAR-SFETD) et concernant l'anesthésie locorégionale en douleur chronique
}

\section{The history and aim of recommendations formalised by common experts from two organisations (SFAR-SFETD) regarding locoregional anaesthesia and chronic pain}

\author{
D. Péronnet \\ (C) Springer-Verlag France 2013
}

En septembre 2011, le conseil d'administration de la Société française d'étude et traitement de la douleur (SFETD) et le comité douleur de la Société française d'anesthésieréanimation (SFAR) décident de réaliser des recommandations d'experts concernant l'utilisation de l'anesthésie locorégionale (ALR) en douleur chronique. Une journée thématique concernant ce sujet a été organisée par la SFETD et le comité douleur de la SFAR le 7 octobre 2011. La méthodologie GRADE et les critères d'efficacité ont été validés par le comité des référentiels de la SFAR en juin 2012 ainsi que la composition du groupe d'experts et les principaux domaines des recommandations choisies.

Ces recommandations formalisées d'experts ont été validées par le conseil d'administration de la SFAR le 19 janvier 2013 et par le conseil d'administration de la SFETD le $1^{\text {er }}$ mars 2013. Elles ont été présentées au congrès national de la SFETD à Lille le 22 novembre 2012. Elles seront présentées au congrès national de la SFAR à Paris le 21 septembre 2013.

Dans ce numéro de Douleur et Analgésie, nous présentons les principales communications de la journée théma- tique douleur du 7 octobre 2011 et les recommandations formalisées d'experts concernant l'ALR en douleur chronique, également publiées sur le site de la SFAR et dans la revue des AFAR.

Le comité de pilotage espère que ces différents médias permettront une large diffusion de ces recommandations, tant dans le milieu très pluriprofessionnel de la douleur que dans la communauté des anesthésistes-réanimateurs. Ces recommandations ont également permis de réaliser un lien entre les deux sociétés savantes.

Ces recommandations devraient servir de support aux praticiens en douleur chronique lorsqu'ils sont confrontés à des problèmes de douleurs rebelles ou de prise en charge globale antalgique mal tolérée ou inefficace. Les praticiens d'anesthésie-réanimation sollicités pour la réalisation de blocs analgésiques verront dans ces recommandations une justification des procédures demandées et validées par les deux sociétés savantes.

Le comité d'organisation : Hélène Beloeil, Éric Viel, Marie-Louise Navez, Dominique Fletcher, Denis Péronnet. 